Itinerarios culturales y rutas turístico-culturales 09

\title{
Rutas patrimoniales en bicicleta. Otro modo de reconocer paisajes de la memoria
}

Carlos Villar Díaz. Arqueólogo y museólogo. Club EnRuta

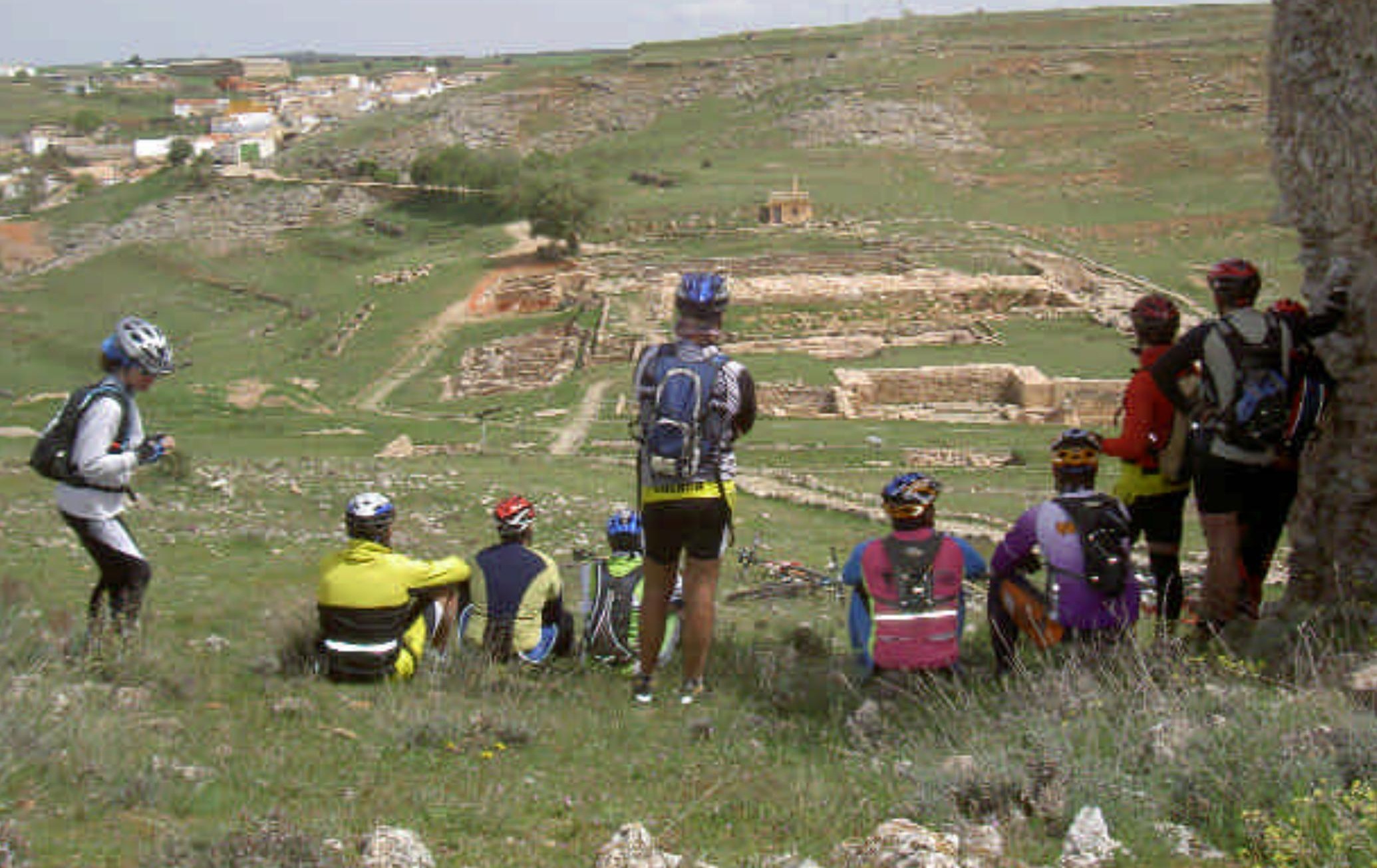


Desde la experiencia que viene adquiriendo nuestra asociación deportiva sin ánimo de lucro ( $w$ w w sercuenca.com/enruta) en la realización de los Itinerarios Culturales y Rutas Histórico Culturales -o grandes travesías, tal como las denominamos en el sector- por todo el ámbito peninsular, hemos comprobado y estamos de acuerdo, cómo todas hilan varios carretes para darse sentido, razón y validez, teniendo siempre como sustrato un movimiento, una acción humana desarro llada en un territorio durante un cierto tiempo y cuyo estímulo fueron relaciones de todo tipo, creando durante el proceso un paisaje diverso -incluso ficticio, como también hemos comprobado muy cerca de nuestro ámbito cercano-, al que se incorporan -objetiva y/o de forma dirigida o canalizada, pero en muchos casos, no cualificada..: la llamada de atención o señalización sobre ciertos elementos identificativos (nodos, polos o waypoints) que sirven de atracción, reconocimiento 0 justificación y no siempre, de generación coherente de múltiples recursos socioeconómicos y culturales. $Y$ hacia ese fin han ido generalizándose por todas las comunidades autónomas, vinculándose más al producto -en su aspecto externo o rentabilidad periférica- y a la moda en su mayoría ${ }^{1}$ que al contenido diversificado y renovado.

Entre los más de setenta socios y colaboradores -afortunadamente ya de ámbito nacional- hemos comprobado que rara vez se repite una gran travesía, incluso aquéllas que podríamos considerar "clásicas "2 a pesar de la propia of erta natural y especialmente climatológica, que nos cambia por completo el escenario de cada ruta, porque en cada experiencia se agota el combustible que lo alimenta, el elemento clave, imprescindible, fundamental y básicamente humano: el interés por lo desconocido junto a la sensación de placer en el proceso de descubrimiento fuera de su medio habitual, la propia naturaleza cer-

Q Observando la ciudad romana de Valeria (Cuenca) / PAblo Fernández cana (de ahí el éxito de los deportes de riesgo controlado..) y su redescubrimiento, que no hace sino acercarnos más a ella para entenderla y respetarla.

Cuando realizamos y/o diseñamos recorridos novedosos - de una sola 0 varias jornadas- desde la versatilidad y complementariedad temática -gracias a la multidisciplinar formación de nuestro grupo y siempre con la colaboración y participación de residentes y vecinos a nuestras "aventuras" como enlaces o nexos al conocimiento cercano o vernáculo- buscamos satisfacer esas cualidades innatas al ser humano, tanto para el natural que se reconoce en su territorio como para el foráneo que descubre otros paisajes. En su renovación encontramos su validez y pervivencia. Pero vamos más allá. Creemos y consideramos que la acción humana debe ser ordenada; no hay más remedio si buscamos que el legado medioambiental -en el que se inserta el cultural solidaria e intrínsecamente- sea reconocido, valorado y disfrutado ahora y en generaciones venideras y por ello, nuestra situación actual nos posiciona ya muy cercanos a la figura del voluntariado medioambiental y cultural, como primer paso previo a la acción $n^{3}$. De hecho, utilizamos la difusión e interpretación de conocimientos in situ, sobre los enclaves por los que transitamos mediante los sopof tes de información disponibles y tributarios de los Parques Naturales o de la Red de Senderos con cuya organización compartimos la práctica totalidad de objetivos y acciones. Y cuando aquéllos se han requerido para nuestras propuestas hemos procurado incluir elementos compatibles con el medio, ya que ante las nuevas tecnologías de la información y la amplia aceptación de los "GPS" o navegadores ${ }^{4}$, se hace cada vez menos necesaria la profusión señalética y porque igualmente seguimos volcándonos hacia la utilización -ahora ya con otra función- de recorridos históricos, que tuvieron sentido una vez y que ahora recuperamos de la forma más respetuosa que alcanzamos, huyendo siempre de los tópicos y las explicaciones fáciles. 


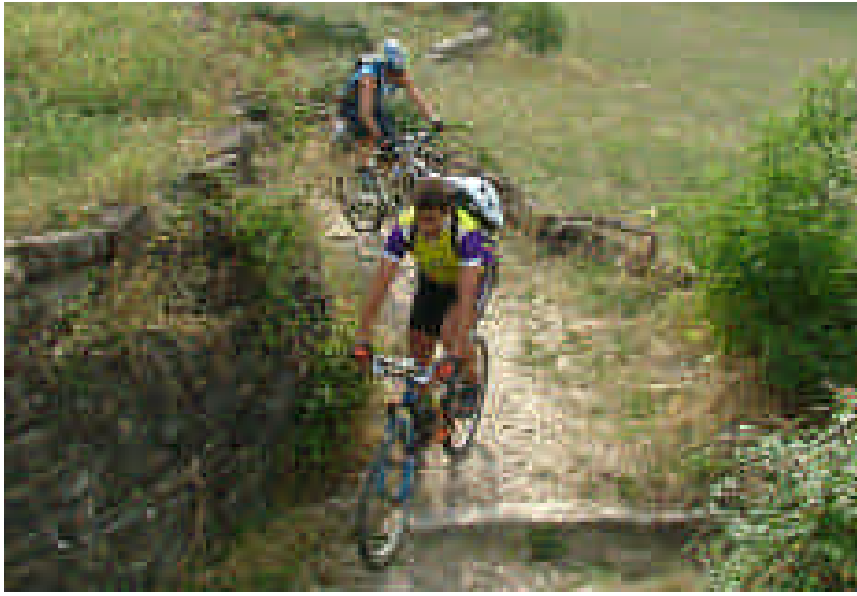

W Senda Pirenaica. Camino de Urdués (Huesca) / CARLos Villar

\section{Intentamos que nuestros socios y todos aquéllos que lo deseen reconozcan, entiendan y valoren los paisajes y territorios cercanos y lejanos}

Practicando uno de los deportes más saludables que existen, la bicicleta de montaña, intentamos que nuestros socios y todos aquéllos que lo deseen, reconozcan, entiendan y valoren los paisajes y territorios cercanos y lejanos que vamos grabando en nuestra memoria - o rescatandoy cómo los elementos más significativos, las claves de su pervivencia 0 los riesgos de su desaparición se interrelacionan y adquieren sentido, ya sean tangibles o no. Es muy significativo comprobar periódicamente cómo ante las heridas, las huellas crueles que dejan las mutaciones en el paisaje, éste se of rece en tenaz resistencia a verse dominado, a brindar innumerables sorpresas y a facilitar opciones de uso -y aquéllos recorridos históricos o tradicionales son los primeros elementos que surgen como enlaces diacrónicos-, aprovechamiento y satisfacciones a quien lo transita y busca posicionarse en ese territorio de un modo respetuoso, integrado, humano.

Y lo más satisfactorio es sin duda, la acción participativa y consecuente sobre el medio, la actitud de crítica positiva comparativa y constructiva y las relaciones humanas conseguidas mediante intercambios de documentación y experiencias. Afortunadamente no estamos solos, compartimos inquietudes con numerosos clubes ya a nivel nacional, y no podemos ignorar que se está produciendo un considerable aumento de interés -solo hay que dar un paseo por la

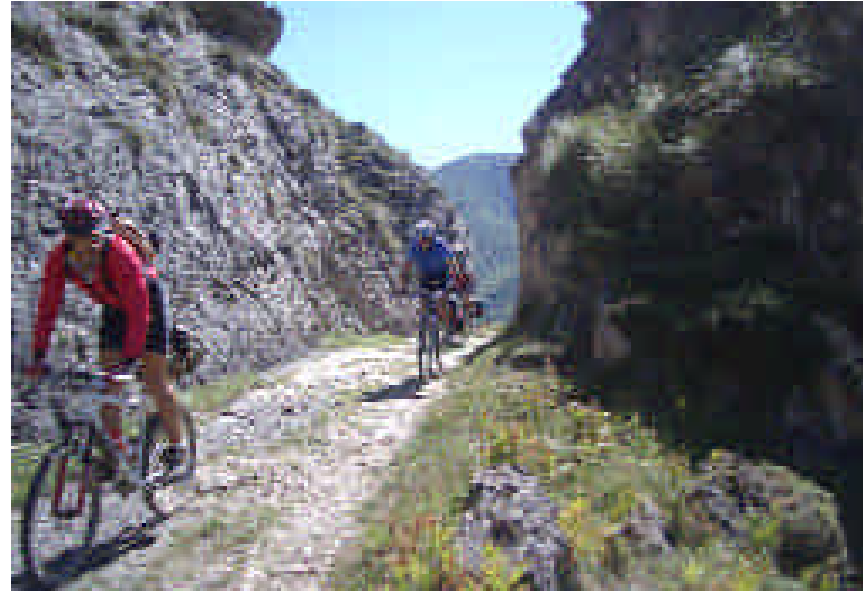

W Itinerario Lebaniego (Collado Panderruedas - Loma Los Carros) / César Colomer

red virtual $u$ of ertar una nueva ruta para observar el panorama- por este particular proceso de reconocimiento o producto si así preferimos denominarlo. Es evidente que éste no es exclusivo puesto que la base deportiva siempre está presente y nunca se elimina -ni lo deseamos- el componente competitivo de la naturaleza humana cuando se realiza en grupo e incluso individualmente, pero a la que si añadíamos el valor complementario de incluir sectores con cierto contenido "extra", ya sea cultural, natural, etc. obteníamos una amplia respuesta participativa. Así nos ocurrió en numerosas ocasiones, incluso de forma no premeditada, de modo que decidimos hace tiempo que no podíamos usar el medio sin devolverle algo a cambio. Y comenzamos a planificar una ruta "especial", mensualmente, una jornada destinada a dejarnos llevar sorpresas por los diversos paisajes y a conocerlos profundamente, en fin a recuperar nuestra memoria cercana, de nuestros propios abuelos. No fue tarea complicada, nuestro territorio es especialmente generoso.

Pero además, hemos encontrado en estos últimos años verdadera pasión por descubrir las rutas e itinerarios sobresalientes de la península y los recorridos más arraigados en la memoria colectiva; desde la exigente Jacetania y sus escondidas joyas arquitectónicas y senderos como el de los Cátaros, a las sorprendentes Alpujarras y los posibles enlaces con las torres de costa de las playas almerienses; desde las resistentes "brañas" asturianas en el Camí de la Mesa y la Vía Carisa a los enlaces trashumantes de la sierra onubense - iy sus tesoros gastronómicos!-; del estepario telón de fondo fosilizado de encinas que asemejan al famoso Alonso Quijano a las Fuentes del Padre Tajo entre agrestes enclaves indígenas prerromanos aún por identificar; de las localizaciones de posibles santuarios y estaciones rupest res mediterráneas -que parece comienzan a ser comprensibles desde estas lecturas del paisaje- a las inverosímiles fortificaciones de enclaves espectaculares y sus claves territoriales fronterizas; de los apacibles agostaderos 


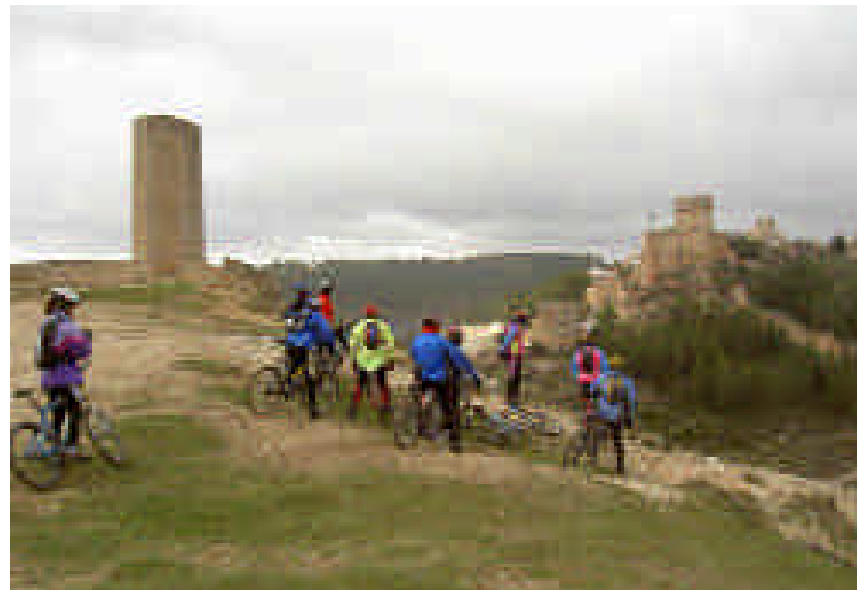

W Explicación ante la Fortificación de Alarcón, desde la Torre del Campo (Cuenca) / Pablo Fernández

serranos a los tradicionales vados, lavaderos y cortaderos solapados e intencionadamente mimetizados con los antiguos puentes, pozos y fuentes romanas...

Ahora ya, ante la respuesta obtenida por nuestros talleres y acampadas destinados tanto para niños y jóvenes en familia como a grupos de educación secundaria, por las publicaciones -guías, programaciones, calendarios y crónicas, archivos de tracks, etc.- y los nuevos proyectos

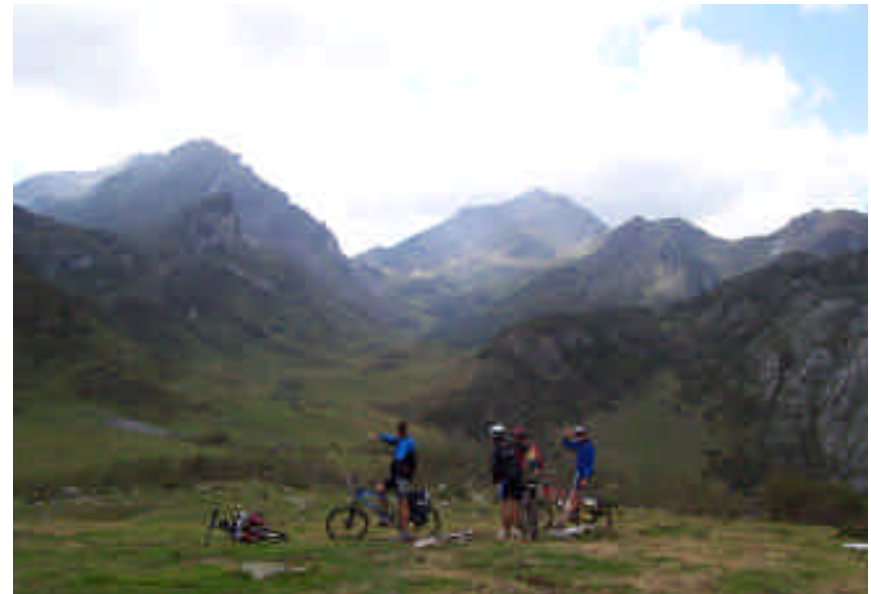

W Buscando el paso natural a León en el Valle del Arcenorio / César Colomer

en preparación $n^{5}$, estamos convencidos de que nuestras próximas acciones (siempre desde el ámbito asociativo, lo que nos permitirá rodar fuera de cualquier presión mercantilizada) más que para la "defensa", seguirán encaminadas hacia la "comprensión" del territorio y su paisaje, de su patrimonio natural y cultural desde estos recorridos, interpretativos si así preferimos llamarlos, y supondrán una contribución a la sostenibilidad de nuestro medio ambiente (aunque se encuentre intensamente antropizado).

\section{Notas}

\begin{abstract}
${ }^{1}$ Precisamente éste es un fenómeno que venimos observando en aquellos itinerarios diseñados desde o con un fuerte control de las instituciones responsables 0 competentes y que igualmente hemos sufrido ante la propuesta de redacción de determinadas rutas fuera de entornos "conflictivos". Pero sería injusto no destacar que existen en el sector esfuerzos muy valorados y reconocidos para ofrecer alternativas atractivas y sugerentes a itinerarios básicos, entre las que sobresale la edición anual fuera de serie Rutas Bike a fondo del grupo MPIB y las guías monográficas de editoriales como Prames, Tutor, Penthalon, etc.

${ }^{2}$ Como el Camino de Santiago, la Ruta de la Plata, El Camino del Cid, etc. y en cuyo sentido originario debiéramos experimentar y así programamos en alguna ocasión, que no sólo consisten en el tránsito de ida sino en la vuelta al lugar de origen, recuperando el concepto del homo viator medieval, mas cercano quizás, a nuestros lejanos ancestros nómadas. También las Vías Verdes raramente se repiten, aunque tienen una gran aceptación por otro tipo de usuarios, si no es para servir de enlaces a otros itinerarios (la Senda del Oso y la Ruta de la Plata en Asturias, o el Ferrocarril de los 40 días y la Cañada Real Soriana, etc.) quizás porque transmiten un cierta sensación de camino readaptado donde no existe un contacto más cercano y real, tangible al propio relieve. La bicicleta es un deporte
\end{abstract}

duro y con la de montaña no existe más limitación que la propia, los que aprendemos a disfrutar de ella rara vez la abandonamos.

${ }^{3}$ Desde la asociación consideramos y creemos firmemente que la ordenación y planificación territorial debe realizarse de un modo más participativo, puesto que el esfuerzo y el tiempo dedicado a la conservación del patrimonio medioambiental de forma desinteresada responde a una inquietud intrínsecamente humana de respeto por el medio que lo acoge, indicando un grado social y de desarrollo que a su vez reconoce el valor de la identidad a su territorio, como un recurso limitado. Consecuentemente, nos hemos ofrecido a la Consejería de Medio Ambiente de la Junta de Comunidades de Castilla La Mancha a facilitar todo el material técnico recopilado en los últimos años y a participar en la redacción y ejecución de acciones propias y específicas del futuro PORN de la Serranía de Cuenca, teniendo como premisa la conocida frase "piensa globalmente, actúa localmente" para mantener inseparable la pervivencia de territorio y paisaje y no desvirtuar el desarrollo rural con falsas esperanzas.

\footnotetext{
${ }^{4}$ Pero sin abandonar el contacto y el uso frecuente del rutómetro-guía en formato texto y cartografía adaptada y recomendar la experiencia de leer el territorio y paisaje, el
} "GPS" es una herramienta y el navegador es la persona.

\footnotetext{
${ }^{5}$ Se acaba de concluir la fase de documentación previa, evaluación y análisis de recursos del territorio que comprende la comarca denominada Campisierra, en la provincia de Cuenca, en colaboración con el Grupo de Acción Local, para la realización de tres itinerarios básicos transversales, a experimentar en el primer semestre del próximo año, que permitan la realización de rutas temáticas complementarias, diversificadas y versátiles tanto para bicicleta de montaña y senderismo, en las que consideramos se complementa el sentido de territorio museo y avanzan conceptos novedosos como la accesibilidad a los nodos -en la que nuestra comunidad ha sido pionera- $\mathrm{y}$ la usabilidad de las herramientas de información. Evidentemente, tanto la planificación de los trabajos previos como la vinculación al medio del equipo de técnicos y colaboradores desinteresados supone ritmos de producción y rendimientos muy diferentes a los of recidos por empresas especializadas con las que ni existe, ni se han producido hasta el momento roces o choque de intereses, dada la naturaleza del objetivo primario, la elaboración de un soporte o red de acceso a determinados recursos. Igualmente nos encontramos en la fase de planificación previa para el año 2007 acometer la revisión y adaptación de varios sectores alcarreños de la Ruta de la Lana, de Cuenca a Burgos, recuperada por Jesús H. Pareja Pérez y Vicente Malabia Martínez y editada en Cuenca por Alfonsípolis en 1999.
} 